\title{
The impact of hypoxia on oncolytic virotherapy
}

\author{
This article was published in the following Dove Press journal: \\ Virus Adaptation and Treatment \\ I5 November 2011 \\ Number of times this article has been viewed
}

\section{Z Sheng Guo}

University of Pittsburgh Cancer Institute and Department of Surgery, University of Pittsburgh School of Medicine, Pittsburgh, PA, USA
Correspondence: Z Sheng Guo University of Pittsburgh Cancer Institute, 5II7 Centre Avenue, Suite I.46, Pittsburgh, PA 15213, USA

Tel +I 4I2623 77II

Fax + I 4I26237709

Email guozs@upmc.edu
Abstract: The hypoxic tumor microenvironment plays significant roles in tumor cell metabolism and survival, tumor growth, and progression. Hypoxia modulates target genes in target cells mainly through an oxygen-sensing signaling pathway mediated by hypoxia-inducible factor of transcription factors. As a result, hypoxic tumor cells are resistant to conventional therapeutics such as radiation and chemotherapy. Oncolytic virotherapy may be a promising novel therapeutic for hypoxic cancer. Some oncolytic viruses are better adapted than others to the hypoxic tumor environment. Replication of adenoviruses from both groups B and C is inhibited, yet replication of herpes simplex virus is enhanced. Hypoxia seems to exert little or no effect on the replication of other oncolytic viruses. Vaccinia virus displayed increased cytotoxicity in some hypoxic cancer cells even though viral protein synthesis and transgene expression were not affected. Vesicular stomatitis virus replicated to similar levels in both hypoxic and normoxic conditions, and is effective for killing hypoxic cancer cells. However, vesicular stomatitis virus and reovirus, but not encephalomyocarditis virus, are sensitive to elevated levels of hypoxia-inducible factor- $1 \alpha$ in renal cancer cells with the loss of von Hippel-Lindau tumor suppressor protein, because elevated hypoxia-inducible factor activity confers dramatically enhanced resistance to cytotoxicity mediated by vesicular stomatitis virus or reovirus. A variety of hypoxia-selective and tumor-type-specific oncolytic adenoviruses, generated by incorporating hypoxia-responsive elements into synthetic promoters to control essential genes for viral replication or therapeutic genes, have been shown to be safe and efficacious. Hypoxic tumor-homing macrophages can function effectively as carrier cells to deliver an oncolytic adenovirus to the hypoxic/necrotic areas of the tumor. It is envisioned that further improved oncolytic viruses will be highly effective against hypoxic tumor, especially when combined with other therapeutic regimens such as immunotherapy, radiation therapy, and/or chemotherapy.

Keywords: hypoxia, oncolytic virus, viral replication, viral spread, oncolysis, HIF, macrophage, combination strategy

\section{Introduction}

The utilization of viruses for cancer treatment has garnered interest for several decades. ${ }^{1}$ These viruses, either by innate properties per se or modified via genetic engineering, possess the ability to infect and/or replicate exclusively in cancer cells, allowing normal cells to remain unharmed. ${ }^{2-4}$ As the first-generation oncolytic viruses (OVs) have been tested in clinical trials, novel genetic engineering approaches have permitted the design of the next generation of recombinant viruses; these OVs demonstrate increased oncolytic potency in neoplastic tissue without compromising safety in animal models. ${ }^{2,5}$ In both preclinical models and clinical trials, OVs have often been utilized 
in combination with radiation therapy, classic chemotherapeutics, prodrug therapy, or immunotherapy in order to achieve better efficacy. ${ }^{6-10}$

Since the first genetically engineered virus, a herpes simplex virus (HSV), was explored as an OV, hundreds of other engineered viruses or naturally selected viruses have been studied in tumor models in preclinical studies. Several of these OVs have been examined in phases I-III clinical trials (for a list of OVs in clinical trials, see the table by Rowan, 2010). ${ }^{11}$ OVs expressing the cytokine granulocyte-macrophage colonystimulating factor have shown great promise. OncoVEX GMCSF (cytokine granulocyte-macrophage colony-stimulating factor) has been tested in phase I/II trials. ${ }^{12}$ A prospective, randomized phase III clinical trial in patients with unresectable stages III and IV melanoma is being completed. ${ }^{13}$ An oncolytic poxvirus expressing cytokine granulocyte-macrophage colony-stimulating factor, JX-594, is genetically engineered for replication and transgene expression in cancer cells harboring an activated epidermal growth factor receptor/Ras pathway, followed by cell lysis and anticancer immunity. The virus, either alone or in combination with sorafenib, a small molecule inhibitor of B-Raf and vascular endothelial growth factor (VEGF) receptor, was well-tolerated and displayed objective tumor response in a small number of patients with hepatocellular carcinoma. ${ }^{14}$ In a recent study, Breitbach et al showed in a clinical trial that JX-594 selectively infects, replicates, and expresses transgene products in cancer tissue after intravenous infusion in a dose-related fashion in human cancer patients. ${ }^{15}$ China approved the world's first OV (H101) for cancer treatment in 2005, showcasing the potential of OVs as a new class of pharmaceutical drugs for cancer patients. ${ }^{16}$

Tumor microenvironment (TME) plays an important role in dictating not only the replication but also oncolytic effects of OVs. ${ }^{3,17-19}$ Hypoxia is one of the hallmarks of solid tumor. The interplay of hypoxic TME and OV is a key factor determining not only the outcome of viral replication, spread, and potential oncolysis in a specific tumor but also the overall efficacy of oncolytic virotherapy. ${ }^{3,17-19}$ The field of oncolytic virotherapy for hypoxic tumor was reviewed by Hay in $2005 .^{20}$ Here, the focus will be on recent new and exciting progress in the field and a discussion of future directions for developing OVs in the setting of treating hypoxic tumors.

\section{Overview of the biological properties of OVs}

Ideally, an OV is a virus that preferentially infects and lyses cancer cells while leaving normal cells unharmed.
These viruses have been explored to treat cancer, both by direct destruction of the tumor cells, and, if modified, as vectors enabling genes expressing anticancer proteins to be delivered specifically to the tumor site. ${ }^{1-3}$ In general an OV is able to attach to and infect the tumor cells, replicate in these tumor cells, and "lyse" them, thereby releasing progeny virus into the surrounding TME and infecting neighboring tumor cells. This process would prime the host immune system and generate specific immunity against the tumor cells as well as the virus. Several recent reports have presented evidence of synergistic effects between direct virus-mediated oncolysis and the activation of specific antitumor immune responses..$^{21,22}$ In the clinical setting, more rational, potentially synergistic combination strategies are being tested. ${ }^{23}$ These combination strategies offer an exciting outlook for the future of cancer virotherapy.

There are some naturally occurring OVs that selectively kill tumor cells while leaving normal cells unharmed. ${ }^{24}$ Examples of these OVs include measles virus, Newcastle disease virus, reovirus, and vesicular stomatitis virus (VSV), most of which are ribonucleic acid (RNA) viruses. The mechanisms of tumor cell selectivity appear to be that these viruses are able to exploit differences in the biology between tumor cells and their normal counterparts that arise during the transformation into a cancer cell. ${ }^{24}$ For example, the selectivity of VSV strains and myxoma virus is mediated by tumor cell-specific defects in interferon responsiveness or innate immunity. ${ }^{24-27}$ Instead, the oncolytic specificity of Newcastle disease virus is selected for apoptosis-resistant cells, not for defect of interferon responsiveness as believed previously. ${ }^{28}$

Most OVs are generated via genetic engineering for enhanced tumor selectivity. Normally, viruses will infect normal cells. For adenoviruses (Ads), this is via an oral or nasal route of entry and involves the epithelial lining of the nose, throat, and/or gut resulting in a respiratory and/or gastrointestinal infection. When used as an agent in cancer therapy, the objective is to develop recombinant Ads that selectively infect a vastly different set of cells, namely, transformed epithelial cells and tumor-associated endothelium located in distinct locations throughout the body, most of which are not normally seen during the typical Ad infection. Researchers must develop OVs that efficiently and selectively replicate in, and kill, cancer cells to which they would not normally be exposed. An additional requirement is that these OVs can adapt to hypoxic tumor cells for survival, to proliferate, and to exert their potential for oncolysis. There are at least two methodologies to develop OVs targeting hypoxic tumor environment. The primary one is based on rational design 
by genetic engineering. An alternative approach employs directed evolution as a means of producing highly selective and potent anticancer viruses. ${ }^{29}$ Using this "directed evolution" methodology, Bauzan and Hermiston generated ColoAd1, a novel chimeric OV. ${ }^{29}$ In vitro, this virus demonstrated a $>2 \log$ increase in both potency and selectivity when compared to ONYX-015 on colon cancer cells. ${ }^{30}$ This approach may be applied to generate other novel and potent OVs targeting hypoxic tumor cells.

\section{Overview of the tumor hypoxic environment and why current therapies do not work effectively}

Hanahan and Weinberg have recently made a new summary of cancer hallmarks. ${ }^{31}$ Adding to the six classic hallmarks are two emerging hallmarks: reprogramming of energy metabolism and evading immune destruction. The key physical and chemical properties of TME often include hypoxia, nutrient deprivation, acidosis, and aberrant stroma. ${ }^{32,33}$ The TME is being increasingly recognized as an important determinant of tumor progression as well as of therapeutic response. ${ }^{34}$ The TME and its tumor-stromal interactions are capable of altering the delivery and effectiveness of therapeutics into the tumor, including oncolytic virotherapy. ${ }^{17-19,34}$

What are hypoxia and tumor hypoxia? As stated by Hockel and Vaupel, ${ }^{35}$ biochemists may define hypoxia as molecular oxygen-limited electron transport. Physiologists and clinicians may define hypoxia as a state of reduced oxygen availability or pressures below critical thresholds, thus hindering or abolishing the function of organs, tissues, or cells. In solid tumors, oxygen delivery to the respiring neoplastic and stromal cells is frequently reduced or even abolished by deteriorating diffusion geometry, severe structural abnormalities of tumor microvessels, and disturbed microcirculation. As a result, areas with very low oxygen partial pressures exist in solid tumors, occurring either acutely or chronically. By definition, there are two types of hypoxia in tumor: chronic hypoxia and intermittent hypoxia. Chronic hypoxia refers to the imbalance between oxygen delivery and oxygen consumption, which exists in regions of tumors beyond the diffusion distance of oxygen. The disorganized tumor neovasculature is generally claimed as the common denominator of the above causes of deficit in oxygen supply. The other form, acute or intermittent hypoxia, is a ubiquitous process occurring within most solid tumors. It can exist at a distance (even more than $100 \mu \mathrm{M}$ ) from a tumor microvessel. Fluctuations in pressure of oxygen of approximately $20 \mathrm{mmHg}$ can occur with periodicities of minutes to hours and even days. ${ }^{36,37}$ The major phenotypic shift associated with chronic hypoxia is tumor cell resistance to chemotherapy and radiotherapy and more invasive and metastatic features. In contrast, acute hypoxia may exert more effects on the phenotype of endothelial cells lining tumor blood vessels, which may have significant therapeutic implications. Acute hypoxia may permit resistance to treatment and thereby affects the survival of hundreds of tumor cells. ${ }^{36}$

Three main oxygen-sensing pathways promote hypoxia tolerance by regulating gene transcription and messenger RNA translation in cells exposed to hypoxia. It has long been known that transcription factors hypoxia-inducible factor- $1 \alpha$ (HIF-1 $\alpha$ ) and HIF-2 $\alpha$ play critical roles in cellular response to hypoxia. ${ }^{38-40}$ The most characterized molecular response to hypoxia is the stabilization and activation of HIF-1. HIF-1 is a heterodimer composed of the HIF- $1 \alpha$ subunit and the HIF-1 $\beta$ subunit. During hypoxia, a shift from aerobic metabolism to glycolytic metabolism is mediated in a large part by HIF-1, via upregulation of various metabolic genes, such as glucose transporters, aldolase, lactate dehydrogenase, and pyruvate kinase. Even though both subunits of HIF-1 are constitutively expressed, HIF- $1 \alpha$ is rapidly degraded in the presence of cellular oxygen via the ubiquitin-mediated proteasome pathway. Recently, two other oxygen-sensitive signaling pathways have also been implicated: signaling via the mammalian target of rapamycin (mTOR) kinase and through activation of the unfolded protein response. ${ }^{41}$ Even though these signaling pathways function independently in response to hypoxia, evidence has accumulated that HIF-, mTOR-, and unfolded protein response-dependent responses to hypoxia act in an integrated way, influencing each other and common downstream pathways that affect gene expression, metabolism, cell survival, tumorigenesis, and tumor growth. ${ }^{41}$ Some recent studies have linked mTOR signal and HIF-1 $\alpha$ to CD133 expression, a cancer stem cell marker in cancer cells. This might lead to insights into the involvement of the mTOR signal and oxygen-sensing intracellular pathways in the maintenance of stemness in cancer stem cells. ${ }^{42-46}$

The other response to hypoxia in mammalian cells is a G1 cell cycle arrest, in addition to adaptive metabolic changes. Early work showed that hypoxia inhibits G1/S transition through regulation of p27 expression. ${ }^{47}$ However, later work showed the real mechanisms are much more complex..$^{48}$ The hypoxia-induced replication arrest initiates a DNA damage response that includes both ataxia telangiectasia mutatedrelated and ataxia telangiectasia mutated plus Rad3-related mediated signaling. ${ }^{49}$ Although G1 cell arrest in response to hypoxia is observed in normal cells and some cancer cells, 
little or no effect on $\mathrm{S}$ phase has been reported in cells with disrupted HIF-1 $\alpha$ signaling and in some cancer cells. G1 arrest of tumor cells in hypoxic conditions may not be critical to limit viral replication for at least some viruses. In fact, hypoxia may induce DNA overreplication in tumor cells. ${ }^{50}$ DNA replication can be blocked by hypoxia, but only at nonphysiological levels of $<0.1 \%$ oxygen. ${ }^{20}$

Hypoxia promotes immune suppression in the TME via multiple mechanisms. It modulates both the innate immunity and adaptive immunity. Hypoxia links to the innate immunity and inflammation via nuclear factor- $\kappa \mathrm{B}(\mathrm{NF}-\kappa \mathrm{B}){ }^{51}$ Monocytes are continually recruited into tumors, differentiate into tumor-associated macrophages, and then accumulate in the hypoxic/necrotic areas. Hypoxia via HIF-1 $\alpha$ dramatically alters the function of myeloid-derived suppressor cells in the TME and redirects their differentiation toward tumorassociated macrophages. ${ }^{52}$ These macrophages exposed to hypoxia upregulate expression of HIF-1 $\alpha$ and HIF-2 $\alpha$, which then bind to hypoxic response elements (HRE) within the promoters of multiple tumor-promoting and adaptive genes to activate their expression. ${ }^{53}$ In a recent study, the authors showed that HIF- $2 \alpha$ modulated macrophage migration by regulating the expression of the cytokine $\mathrm{M}-\mathrm{CSF}$ receptor and chemokine ( $\mathrm{C}-\mathrm{X}-\mathrm{C}$ motif) receptor $4,{ }^{54}$ receptors important for tumor cell proliferation, invasion, and metastasis. ${ }^{55}$ In another study, targeted deletion of HIF-1 $\alpha$ in macrophages in a progressive murine model of breast cancer resulted in reduced tumor growth, although VEGF-A levels and vascularization were unchanged. ${ }^{56}$ The authors found that hypoxia powerfully augmented macrophage-mediated T-cell suppression in vitro via HIF-1 $\alpha$ expressed from macrophages. ${ }^{56}$ These responses may help explain the correlation between high numbers of tumor-associated macrophages and poor prognosis in various forms of cancer and offer a potential target for therapy. ${ }^{57-59}$

Hypoxia can exert direct effects on adaptive immunity. It drives the extracellular accumulation of adenosine in the local TME. The adenosine triggers the immunosuppressive signaling via intracellular cyclic adenosine monophosphateelevating A2A adenosine receptors on antitumor T cells. ${ }^{60}$ In addition, the activated antitumor $\mathrm{T}$ cells in hypoxic TME could be inhibited by elevated levels of immunosuppressive HIF-1 $\alpha$. Recent studies have demonstrated that hypoxia leads to a decrease in cytotoxic $\mathrm{T}$ lymphocyte-mediated tumor cell lysis via the cooperative induction of HIF-1 $\alpha$ and the phosphorylation of signal transducer and activator of transcription 3, and the induction of NANOG in target cancer cells. ${ }^{61,62}$ Another mechanism is that tumor hypoxia also promotes the recruitment of regulatory $\mathrm{T}$ cells through induction of expression of chemokine (C-C motif) ligand 28, which in turn promotes tumor tolerance and angiogenesis. ${ }^{63}$ In summary, hypoxia-induced changes lead to the diminished innate and adaptive immunity against cancer. These results may help explain the poor responses of hypoxic cancer to immunotherapy.

Hypoxia may also lead to resistance to ionizing radiation and chemotherapy by depriving tumor cells of the oxygen essential for the cytotoxic activities of these agents. Hypoxia may also reduce tumor sensitivity to radiation therapy and chemotherapy through one or more indirect mechanisms that include proteomic and genomic changes. ${ }^{64,65}$ Tumor hypoxia influences the outcome of cancer radiotherapy. Oxygen is a potent chemical radiosensitizer. Oxygen is an extremely electron-affinic molecule that participates in the chemical reactions that lead to the production of DNA damage after the absorption of energy from ionizing radiation. Cells that are anoxic during irradiation are about three times more resistant to radiation than cells that are well oxygenated at the time of irradiation. ${ }^{66}$ Because the underlying chemical reactions are essentially complete within a few milliseconds after irradiation, oxygen must only be present during irradiation to produce full radiosensitization. Resistance of hypoxic tumor to chemotherapy comes from multiple mechanisms. Three basic categories underlie chemotherapy failure: inadequate pharmacokinetic properties of the drug, tumor cell intrinsic factors (eg, drug efflux pumps), and tumor cell extrinsic conditions which are characterized by such hostile conditions as hypoxia, acidosis, nutrient starvation, and increased interstitial pressure present in the TME. ${ }^{67}$ For example, Rohwer and colleagues recently identified $\mathrm{HIF}-1 \alpha$ as a potent regulator of p53 and NF- $\kappa$ B activity under conditions of genotoxic stress derived from chemotherapy in gastric cancer. ${ }^{68}$ In summary, multiple molecular mechanisms involving hypoxia contribute to resistance to conventional therapeutics. Despite recent advancement in the knowledge regarding tumor hypoxia, there are still major questions to be addressed if the longstanding goal of exploiting tumor hypoxia is to be realized. To address these questions, investigators have studied therapeutic modalities including prodrugs activated by hypoxia, hypoxia-specific gene therapy, targeting transcription factor HIF-1, and tumor metabolism. ${ }^{40,64,69}$

\section{The effects of hypoxia and leaky vasculature on the access and spread of OVs}

Many OVs are large particles, thus they suffer from inefficient extravasations from tumor blood vessels after 
systemic delivery. Their ability to reach cancer cells is an important consideration in achieving specific oncolytic targeting and potential viral replication. Hypoxia upregulates the expression of vascular permeability factor, VEGF, thus enhancing the vascular permeability. This should promote both extravasation of input OV into tumor cells and spreading of progeny OVs to neighboring tumor cells. However, increased vascular permeability and inflammation will also promote infiltration of immune cells that will prematurely clear the viruses resulting in reduction of therapeutic efficacy.

A number of research laboratories have come up with strategies to modulate tumor vascular leakiness in order to enhance the efficiency of specific delivery of OVs to tumor cells. Kurozumi and colleagues investigated OV therapy-induced changes in tumor blood vessels and the impact of modulating tumor vasculature on the efficacy of OV therapy. ${ }^{34}$ They found that OV treatment increased tumor vascular permeability, host leukocyte infiltration into tumors, and intratumoral expression of inflammatory cytokine genes, including interferon- $\gamma$. Pretreatment with cyclic Arg-Gly-Asp peptide, which is angiostatic, reduced tumor vascular permeability, leukocyte infiltration, and interferon- $\gamma$ protein levels, increased viral titers in tumor tissue, and had longer median survival. ${ }^{34}$ Vile and associates have shown that antiangiogenic cancer therapy combined with oncolytic virotherapy leads to regression of established tumors in mice. ${ }^{70}$ They initially hypothesized that transient destabilization of tumor vasculature by $\mathrm{VEGF}_{165}$ may facilitate intratumoral delivery of OVs. It turned out that, although $\mathrm{VEGF}_{165}$ enhanced vascular leakage, therapeutic effects derived mainly from $\mathrm{VEGF}_{165}$-mediated stimulation of endothelial cells transiently to support viral replication. Appropriately timed systemic virus delivery led to viral replication in and lysis of tumor-associated endothelial cells and innate immune-mediated antivascular effects with subsequent vascular collapse. In another study, Breitbach et al showed that VSV directly infects and destroys tumor vasculature in vivo but leaves normal vasculature intact. VSV replicates in tumor neovasculature and spreads within the tumor mass, initiating an inflammatory reaction including a neutrophil-dependent initiation of microclots within tumor blood vessels. ${ }^{71}$ Tseng et al showed that VEGF and/or metronomic chemotherapy regimens could indeed enhance tumor vascular permeability and directly enhances oncolytic Sindbis virus targeting in tumor models. ${ }^{72}$

\section{The effects of hypoxia on the replication and oncolysis of OVs}

The objective of all viruses is to infect target cells, replicate large numbers of progeny virions, and spread these progeny to initiate new rounds of infection. However, target organisms possess both systemic and cell-based defenses to limit virus infection, including immune and inflammatory processes and the execution or suicide of infected cells. ${ }^{73}$ Protective mechanisms (such as induction of apoptosis) are in place in normal cells to prevent viral replication. In hypoxic cancer cells, the eventual success or failure of viral replication may depend on the interplay between adaptive processes that allow survival of tumor cells in hypoxia and cellular protective responses to inhibit viral replication. A major cellular response to stresses, such as hypoxia and viral infection, is the shutdown of protein synthesis. Hypoxia affects host cell translational machinery. Since all viruses depend on the host translational machinery to translate their own proteins, the hypoxic state of infected cells may play a critical role in the viral life cycle, replication, and thus the success of oncolytic virotherapy. ${ }^{20}$

The rationale is to treat the hypoxic tumor with an OV that can infect, replicate in, and lyse the hypoxic tumor cells. Alternatively, it is possible to make hypoxic tumor cells less hypoxic, or even normoxic, so that these "modified" tumor cells or tumor cells in the "modified" TME are now susceptible to infection, replication, and oncolysis by OVs. How inflammation induced by virus infection impacts on the TME is also an important issue. Breitbach et al showed that administration with VSV and vaccinia virus (VACV) resulted in a dramatic transcriptional activation of the proinflammatory neutrophil chemoattractants (chemokine [C-X-C motif] ligand 1 and 5) and results in neutrophil attraction into the TME. The infiltrated neutrophils in turn contributed to acute reduction in tumor vasculature. ${ }^{74}$ Kirn et al showed that systemically administered VACV resulted in infection and subsequent destruction of tumor endothelial cells, which led to loss of tumor vascular density. ${ }^{75}$ Therefore, treatment with OVs may strengthen hypoxia in certain areas of the tumor.

Nine years ago, Hernandez-Alcoceba and colleagues pioneered the work of genetic engineering of an oncolytic Ad specifically for hypoxic tumor. ${ }^{76}$ Since then, many studies from a number of research laboratories have explored the properties of various OVs for hypoxic tumors. The following section summarizes the main findings regarding some representative OVs in the setting of hypoxic tumors. Some key effects of hypoxic conditions on selective OVs are listed in Table 1.

\section{Ads}

As just mentioned, the pioneering work on engineering an OV for hypoxic tumor was from Clarke and associates 
Table I Effects of tumor hypoxia on oncolytic viruses

\begin{tabular}{|c|c|c|c|c|}
\hline Oncolytic virus & Replication efficiency & Oncolysis & Specific mechanisms & References \\
\hline \multirow[t]{2}{*}{ Adenovirus } & \multirow[t]{2}{*}{ Down } & \multirow[t]{2}{*}{ Down } & I. EI A downregulation & Pipiya et al $^{82}$ \\
\hline & & & 2. GI cell growth arrest & Shen et $\mathrm{al}^{83}$ \\
\hline Hypoxia-dependent & Up & Up & Hypoxic response elements- & Post et $\mathrm{al}^{90}$ \\
\hline \multirow[t]{3}{*}{ adenoviruses } & & & containing promoter to drive & Wang et $\mathrm{al}^{86}$ \\
\hline & & & an essential gene for viral & \\
\hline & & & replication & \\
\hline \multirow[t]{2}{*}{ Herpes simplex virus-I } & \multirow{2}{*}{ Up } & \multirow{2}{*}{ Up } & I. Upregulation of GADD34 & Aghi et al ${ }^{92}$ \\
\hline & & & 2. Targeting p53 cancer cells & Fasullo et $\mathrm{al}^{93}$ \\
\hline Vaccinia virus & Similar & $\begin{array}{l}\text { Enhanced (in some } \\
\text { hypoxic cells) }\end{array}$ & Unknown & Hiley et $\mathrm{al}^{95}$ \\
\hline Vesicular stomatitis virus & Similar & Similar & & Connor et $\mathrm{al}^{96}$ \\
\hline
\end{tabular}

Abbreviation: GADD34, growth arrest and DNA-damage inducible gene 34 .

in $2002 .{ }^{76}$ In that study, they designed an oncolytic Ad in which the early region $1 A(E 1 A)$ gene was controlled by an HRE-containing promoter. The tight control of E1A expression correlated with the ability of the virus to replicate in and kill the human cancer cells that were maintained under hypoxic conditions. ${ }^{76}$ Subsequently, a number of groups have designed other hypoxia-dependent oncolytic Ads or utilized them to treat various types of cancer models. ${ }^{77-80}$ It is worth pointing out that such an OV could be highly efficient for the von Hippel-Lindau (VHL)-deficient tumor where HIF is constitutively elevated because of impaired ubiquitination of this transcription factor. ${ }^{78}$ In summary, these early studies demonstrated in principle that such genetically designed oncolytic Ads could be used, alone or in combination, to treat hypoxic solid tumors.

In 2005, two groups evaluated how hypoxia affects different aspects of Ad biology, including attachment and uptake, transgene expression, and replication, in cancer cells and primary normal cells. ${ }^{81,82}$ Both studies, one by Shen and Hermiston ${ }^{81}$ and the other by Pipiya et al, ${ }^{82}$ found that viral replication was compromised by hypoxic conditions. Hypoxia-induced reduction in E1A levels was mediated at the posttranscriptional level. The combination of reduced E1A protein and hypoxia-induced G1 arrest of cells may be responsible for the lack of efficient viral replication under hypoxic conditions. A further study using group B Ads (types 3 and $11 \mathrm{p}$ ) showed that the lytic potential of these viruses is also compromised in a cell-dependent fashion. This study suggests that both group B and group C (Ad-5-based) Ads need to be modified in order to effectively treat hypoxic components of human tumors. ${ }^{83}$

Recently, investigators have returned to engineer second generation HIF-activated oncolytic Ads that display improved replication, oncolytic, and antitumor efficacy. ${ }^{84}$ These Ads are often armed with a transgene to further enhance its specificity and/or potency. A dual-regulated oncolytic Ad (CNHK500), in which human telomerase reverse transcriptase gene promoter controlling $E 1 A$ gene and hypoxia-responsive promoter controlling $E 1 B$ gene, showed increased safety with preserved antitumoral efficacy. ${ }^{85} \mathrm{In}$ order to improve therapeutic efficacy, many such derived oncolytic Ads are armed with different classes of genes. One such study utilized a gene (wild type p53) for induction of apoptosis ${ }^{86}$ Another group combined the hypoxia-targeted oncolytic Ad with genes encoding HSV thymidine kinase and bacterial nitroreductase for virus-directed enzyme prodrug therapy ${ }^{87}$ Another group armed such an Ad with a gene for VEGF receptor 1-Ig and indeed it displayed a concurrent antiangiogenic effect in a tumor model. ${ }^{88}$ In another study, the gene encoding CD40 ligand was used due to its immunostimulatory activity. ${ }^{89}$ Post et al made an oncolytic Ad armed with an interleukin-4 gene (HYPR-Ad-IL4). The interleukin-4 cytokine was chosen for its ability to induce a strong host antitumor immune response and for its potential antiangiogenic activity. ${ }^{90}$ Finally, in order to enhance targeting specificity of the virus, a tumor-type-specific promoter could be used. One such virus utilized a modified human alpha-fetal protein gene promoter with multiple copies of HRE to stringently control the expression of E1A proteins, thus viral replication. This virus (Ad-HRE $\left.\mathrm{A}_{12} / \mathrm{hAFP} \Delta 19\right)$ is a promising agent for hepatocellular carcinoma. ${ }^{91}$

\section{HSV}

Two studies have demonstrated the enhanced replication of oncolytic HSVs by hypoxia. ${ }^{92,93}$ Aghi et al showed that hypoxic glioblastoma U87 cells yielded 4\% more wild-type HSV and 3.6-fold more oncolytic HSV G207 after 48 hours of infection when compared with normoxic cells. ${ }^{92}$ Reverse transcriptase polymerase chain reaction analysis confirmed a five-fold hypoxia-induced upregulation of GADD34 
(growth arrest and DNA-damage inducible gene 34) messenger RNA, a factor complementing the $\gamma 34.5$ gene deletion in G207. In vivo, G207 also exhibited enhanced replication in hypoxic environments, partly due to increased GADD34 expression in hypoxic cells. ${ }^{92}$ In the second study, Fasullo et al showed that another oncolytic HSV, R3616, which lacks the neurovirulence factor infected cell protein 34.5 (ICP34.5), may target hypoxic breast cancer cells that lack functional p53. ${ }^{93}$ However, this conclusion was based solely on the comparison of viral yields from hypoxic and normoxic MDA-MB-231 (p53-) and MCF-7 (p53+) cells, and more data are needed to confirm that such enhanced viral replication depends only on p53 status. In summary, the unique tropism of oncolytic HSVs for hypoxic tumor environment contrasts with the hypoxia-mediated impairment of standard therapies; this enhances HSV's appeal and efficacy in treating such hypoxic cancers as glioblastoma, pancreatic, cervical, and breast cancer.

Another strategy of targeting oncolytic HSV to hypoxic tumor, as done with Ads, is to exploit the differential activation of HIF-dependent gene expression in tumors versus normal tissue. ${ }^{94}$ Post and associates worked on such a strategy by placing an essential gene for viral replication, ICP4, under the regulation of an HIF-responsive promoter and then introduced into the thymidine kinase locus $\left(\mathrm{U}_{\mathrm{L}} 23\right)$ of HSV d120, which contains partial deletions in the two endogenous ICP4 genes. Unexpectedly, HIF-HSV expressed ICP4 and induced tumor cell lysis at similar levels under normoxia and hypoxia. They found out that the lack of HIF-dependent ICP4 transgene expression by HIF-HSV was due to two factors: reversion of the ICP4 gene region to its wild-type configuration and increased HIF-transcriptional activity under normoxia when cells were infected with any strain of HSV-1. The findings have important implications for applications of this oncolytic HSV in cancer therapy and this genetic engineering strategy to other OVs targeting hypoxic tumor.

\section{VACV}

Hiley and colleagues explored the use of an oncolytic VACV for hypoxic tumors. ${ }^{95}$ They examined the Lister strain VACV in a panel of pancreatic cancer cells after exposure to normoxic or hypoxic conditions. Viral protein production and transgene expression were not affected by hypoxia. Interestingly, there was a 3.5-fold and 20-fold increase in viral cytotoxicity for CFPac1 and MiaPaca2 cell lines, respectively, in hypoxic conditions. Cytotoxicity was equivalent in the remaining cell lines. This study suggests that VACV is a promising vector for targeting pancreatic cancer and potentially other hypoxic tumor types..$^{95}$ It would be of interest to determine the mechanisms of enhanced cytotoxicity of the virus in the two cell lines under hypoxia.

\section{VSV}

VSV, an RNA OV, is promising as its replication is naturally targeted to cancer cells with defects in innate immunity. ${ }^{25,26}$ In an early study, Connor and colleagues demonstrated that VSV is capable of replication in cancer cells under hypoxic conditions. ${ }^{96}$ In hypoxia-stressed cells, VSV infection produced larger amounts of messenger RNA than under normoxic conditions. However, translation of these messenger RNAs was reduced at earlier times postinfection in hypoxiaadapted cells than in normoxic cells. Surprisingly, at later times postinfection, the virus overcame a hypoxia-associated increase in alpha subunit of eukaryotic initiation factor-2 phosphorylation and initial suppression of viral protein synthesis to produce large amounts of viral proteins. VSV infection caused the dephosphorylation of the eukaryotic translation initiation factor-4E and inhibited host translation similarly under both normoxic and hypoxic conditions. VSV progeny virus was produced at similar levels in both hypoxic and normoxic cells. As expected, virus infection induced classical cytopathic effects and apoptotic cell death. In vivo, VSV infected and replicated in hypoxic areas of tumors in nude mice. These results show for the first time that VSV has an inherent capacity for infecting and killing hypoxic cancer cells. This ability could represent a critical advantage over existing therapies in treating established tumors. Interestingly, HIF promotes the expression of the interferon- $\beta$ and other genes with antiviral activity upon viral infection. ${ }^{97}$ It has been known that VHL tumor suppressor protein targets HIFs for oxygen-dependent proteolysis. ${ }^{98}$ Therefore, in VHL mutated renal cell carcinoma (RCC) cells, HIFs are highly elevated. As a result, VSV, and possibly other viruses as well, may not be suitable for treatment of RCC with mutated VHL tumor suppressor protein. In those VHL-/- tumor cells, elevated HIF activity induced by hypoxia confers dramatically enhanced resistance to VSV-mediated cytotoxicity. ${ }^{97}$

It is worth noting that VSV infection can generate complicated immune consequences. Intratumoral VSV induced a transforming growth factor- $\beta$-dependent suppressive activity mediated by $\mathrm{CD} 11 \mathrm{~b}(+) \mathrm{GR}-1(+)$ cells that significantly inhibited both antigen-specific T-cell activation. ${ }^{99}$ VSV interferes with tumor dendritic cell function and blocks tumor antigen presentation. ${ }^{100}$ Therefore, adjustments need to be made if VSV treatment is combined with other types of immunotherapy regimens. 


\section{Reovirus}

Cho et al tested how oncolytic reovirus and its target cells would respond to hypoxia. ${ }^{101}$ They found that reovirus infection suppresses HIF-1 $\alpha$ at the protein level, but not at the messenger RNA level, in colon cancer HCT116 cells under hypoxic conditions. This reduction of HIF- $1 \alpha$ is independent of VHL or p53 because it took place in both VHL-/- renal carcinoma A498 and p53-/- HCT116 colorectal cancer cells. However, treatment with the inhibitor MG132 restored HIF-1 $\alpha$ levels, suggesting that reovirus-induced HIF-1 $\alpha$ decrease requires proteasomal activity. The authors proposed to use reovirus together with an HIF-1 $\alpha$ inhibitor as a potential therapeutic regimen against chemoresistant or radioresistant tumors that are hypoxic with increased levels of HIF- $1 \alpha{ }^{101}$ As a result, there appears to be a functional similarity between reovirus and VSV in their sensitivity to elevated levels of HIF-1 $\alpha .^{97,101}$

\section{EMCV}

Picornaviruses have been explored as OVs. Seneca Valley virus-001 is a novel naturally occurring replicating picornavirus with potent and selective tropism for neuroendocrine cancer cell types, including small cell lung cancer. It has been shown to be safe in patients. ${ }^{102}$

Encephalomyocarditis virus (EMCV) is another picornavirus that can function as an OV. As mentioned above, recent evidence has shown that HIF increases NF- $\mathrm{KB}-$ mediated antiapoptotic response in clear-cell RCC that commonly exhibits hyperactivation of HIF due to the loss of its principal negative regulator, VHL. In a recent study, ${ }^{103}$ Roos et al showed that EMCV challenge induces a strong NF- $\kappa \mathrm{B}-$ dependent gene expression profile concomitant with a lack of interferonmediated antiviral response in VHL-null RCC. Established RCC cell lines and early-passage primary RCC cultured cells were acutely susceptible to EMCV replication and virulence.
A
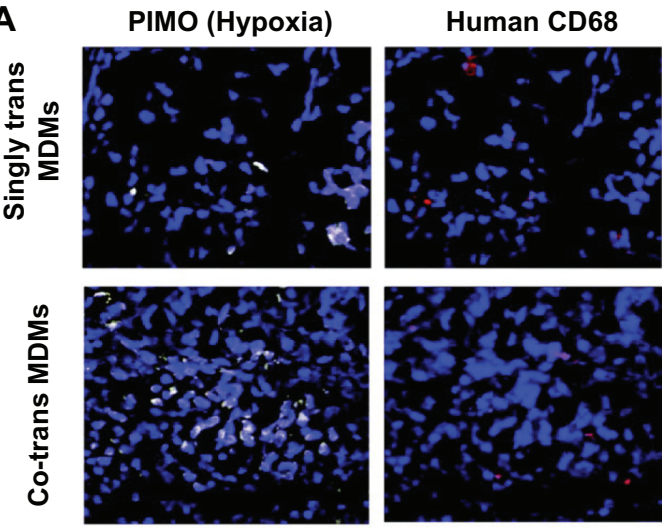

B
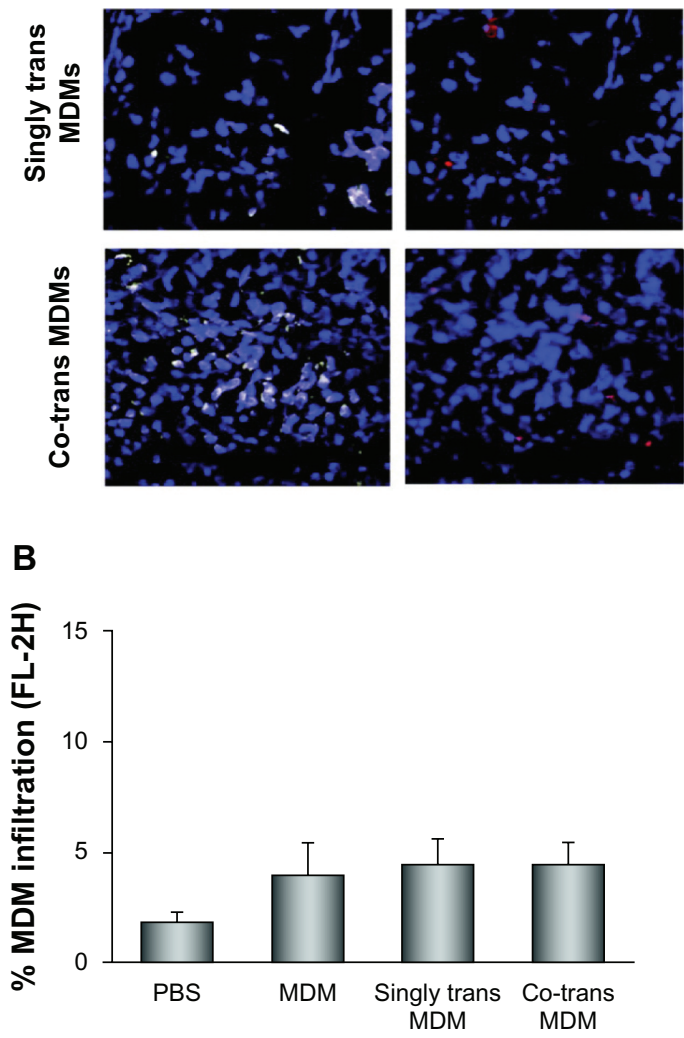

GFP
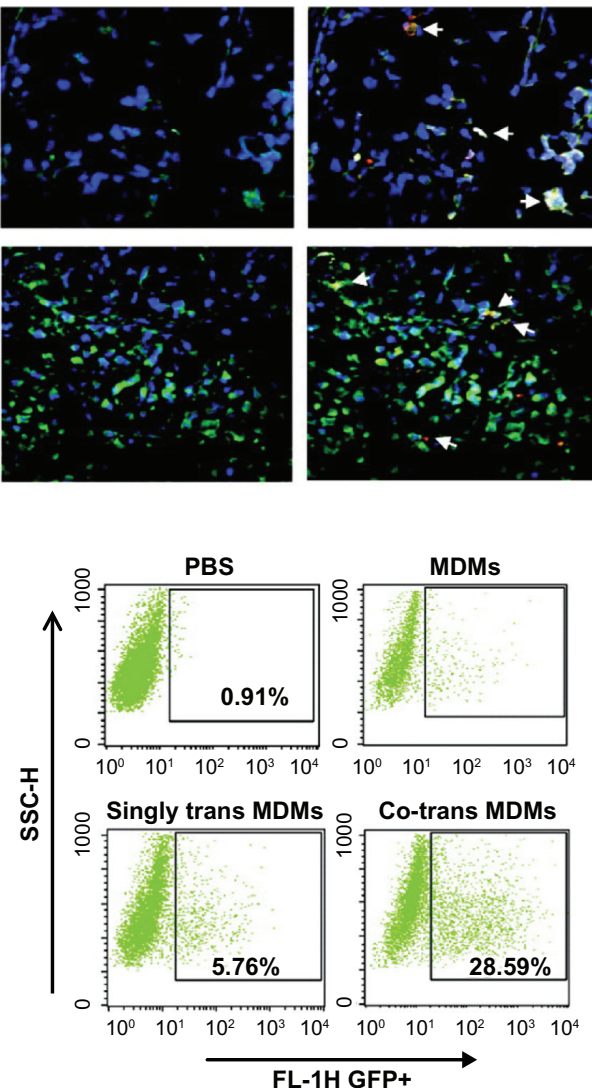

Figure I Cotransduced (co-trans) monocyte-derived macrophages (MDMs) release green fluorescent protein (GFP)-expressing adenovirus in hypoxic prostate tumors. Human untransduced, singly transduced (singly trans), or cotransduced MDMs were injected systemically into athymic mice bearing LNCaP xenografts. At 48 hours later, tumor xenografts were removed, sectioned for immunofluorescence, or enzymatically dispersed for flow cytometry. Frozen sections were labeled with antibodies against pimonidazole (PIMO; white), human CD68 (red), and GFP (green). (A) White arrows show hypoxic macrophages. (B) Single cell suspensions were stained with antihuman CDI4PE or propidium iodide and analyzed by flow cytometry. At right are representative fluorescent histograms from the same treatment groups. Data average \pm standard deviation, $\mathrm{n}=5$ mice/group.

Note: This figure is adapted and reprinted by permission from the American Association for Cancer Research: Muthana M, Giannoudis A, Scott SD, et al, Use of macrophages to target therapeutic adenovirus to human prostate tumors, Cancer Research, 20I I, volume 7I issue 5, I805-I8I5. ${ }^{106}$

Abbreviations: FL-IH, intensity (height) in the FL-I channel; FL-2H, intensity (height) in the FL-2 channel; PBS, phosphate buffered saline; SSC-H, side scatter height values. 
Intratumoral injection of EMCV led to rapid regression of tumor growth in a murine RCC xenograft model. This study provides compelling preclinical evidence for the use of EMCV in the treatment of RCC and potentially other tumors

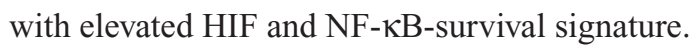

\section{Hypoxic tumor-infiltrating macrophages as carrier cells to deliver OVs}

Macrophages and their precursors in the bloodstream, monocytes, are continually recruited into tumors and migrate into avascular hypoxic/necrotic areas. ${ }^{104,105}$ The fact that tumorassociated macrophages can infiltrate into hypoxic/necrotic areas of tumors prompted investigators to exploit macrophages as a vehicle to deliver HIF-regulated OV and therapeutic genes to otherwise inaccessible areas in tumors. Earlier this year, Muthana et al described a new cell-based method that selectively targets an oncolytic Ad to hypoxic areas of prostate tumors using macrophages as carriers. ${ }^{106}$ Macrophages were cotransduced with a hypoxia-regulated E1A/B construct and an E1A-dependent oncolytic Ad, whose replication is restricted to prostate tumor cells. When such cotransduced macrophages reach an area of extreme hypoxia, the E1A/B proteins are expressed, thereby activating replication of the Ad. This was indeed the case as demonstrated in mice bearing subcutaneous or orthotopic prostate tumors. The cotransduced macrophages migrated into hypoxic tumor areas, upregulated E1A protein, and released multiple copies of progeny Ad. The virus then infected neighboring cells and was cytotoxic in prostate tumor cells, resulting in the marked inhibition of tumor growth and reduction of pulmonary metastases (Figure 1). ${ }^{106}$

This approach is very innovative and efficacious to deliver an oncolytic Ad to hypoxic areas of tumor. However, as correctly pointed out by Baas, it remains unclear whether relying on hypoxic regulation as the only homing determinant will be sufficient to deliver an OV to micrometastatic disease, which are much smaller than solid tumors and not overtly hypoxic. ${ }^{107}$ In addition, macrophages may not function effectively as carrier cells for other OVs. For example, murine primary macrophages are not susceptible to infection by an oncolytic poxvirus (Guo et al, unpublished data).

\section{Conclusion}

Some OVs adapt to the hypoxic environment better than others. Oncolytic HSVs display enhanced replication while Ads show reduced replication potency in hypoxic cancer cells. Other OVs such as VACV and VSV show similar replication efficiency in hypoxic and normoxic conditions. Interestingly, VACV-mediated oncolysis is enhanced in some hypoxic pancreatic cancer cells even though there was no evidence of enhanced viral replication.

\section{Future directions}

To further improve oncolytic virotherapy, the underlying mechanisms through which hypoxia and an OV interact to control life cycles of both host cells and the particular virus in tumor cells need to be understood, and consequently the means to optimize not only viral production but also the virus-induced cytotoxicity in such pathogenic TME need to be developed. The mechanistic studies may lead to further understanding of regulatory signaling pathways for efficient viral replication, eventual oncolysis, and subsequent immunological responses to both the tumor antigens and the virus itself. This will lead to more effective oncolytic virotherapy. In addition, more efficient OVs for hypoxic TME could be obtained via two approaches. The first is rational design via genetic engineering. Alternatively, "directed evolution" methodology may be used. ${ }^{29,30}$ Under this approach, highly diverse viral pools will be placed under stringent directed selection to generate and identify highly potent agents for hypoxic tumors.

More successful therapeutic regimens for hypoxic tumor may lie in the combination of OV with different OVs or other proven modalities such as chemotherapy, radiotherapy, and/or immunotherapy, which has become a research trend for oncolytic virotherapy in the last few years. ${ }^{23,108}$ The strategy of combining oncolytic virotherapy with chemotherapy for treating hypoxic areas of cancer is supported by mathematical modeling. Owen and colleagues applied mathematical modeling and predicted synergistic antitumor effects of combining a macrophage-based, hypoxia-targeted gene therapy with chemotherapy. ${ }^{109}$ Oncolytic virotherapy itself has been considered to be a type of immunotherapy. Evidence is accumulating that it potentially can function synergistically with other types of immunotherapy. ${ }^{110-112}$ The use of carrier cells to deliver OVs to hypoxic areas of tumor has shown early promise. ${ }^{106}$ It is worth exploring further the application of macrophages and other hypoxic tumor-homing cells as carriers for delivering OVs to hypoxic tumor.

An emerging concept in cancer therapy is normalization of tumor vasculation as a complementary therapeutic paradigm. ${ }^{113,114}$ Tumor treated with inhibitors that block epidermal growth factor receptor, Ras, or phosphatidylinositol-3-OH kinase-Akt resulted in prolonged and durable enhancement of tumor vascular flow, perfusion, and decreased tumor hypoxia. ${ }^{114}$ It is tempting to think that, 
following such treatments, even "regular" (not hypoxiatargeted) OVs could be utilized to treat these preconditioned cancers effectively.

\section{Acknowledgments}

I thank Mark O'Malley for English editing. I would like to thank Sunny Zhang for her friendship and tireless encouragement over many years.

\section{Disclosure}

The author reports no conflicts of interest in this work.

\section{References}

1. Kelly E, Russell SJ. History of oncolytic viruses: genesis to genetic engineering. Mol Ther. 2007;15(4):651-659.

2. Cattaneo R, Miest T, Shashkova EV, Barry MA. Reprogrammed viruses as cancer therapeutics: targeted, armed and shielded. Nat Rev Microbiol. 2008;6(7):529-540.

3. Guo ZS, Thorne SH, Bartlett DL. Oncolytic virotherapy: molecular targets in tumor-selective replication and carrier cell-mediated delivery of oncolytic viruses. Biochim Biophys Acta. 2008;1785(2):217-231.

4. Kirn DH, Thorne SH. Targeted and armed oncolytic poxviruses: a novel multi-mechanistic therapeutic class for cancer. Nat Rev Cancer. 2009; 9(1):64-71.

5. Kaur B, Cripe TP, Chiocca EA. "Buy one get one free": armed viruses for the treatment of cancer cells and their microenvironment. Curr Gene Ther. 2009;9(5):341-355.

6. Guo ZS, Parimi V, O'Malley ME, et al. The combination of immunosuppression and carrier cells significantly enhances the efficacy of oncolytic poxvirus in the pre-immunized host. Gene Ther. 2010;17(12): $1465-1475$

7. Harrington KJ, Vile RG, Melcher A, Chester J, Pandha HS. Clinical trials with oncolytic reovirus: moving beyond phase I into combinations with standard therapeutics. Cytokine Growth Factor Rev. 2010; 21(2-3): 91-98.

8. Thorne SH, Liang W, Sampath P, et al. Targeting localized immune suppression within the tumor through repeat cycles of immune cell-oncolytic virus combination therapy. Mol Ther. 2010;18(9):1698-1705.

9. Wongthida P, Diaz RM, Pulido C, et al. Activating systemic T cell immunity against self tumor antigens to support oncolytic virotherapy with vesicular stomatitis virus. Hum Gene Ther. 2011; April 11. [Epub ahead of print.]

10. Mahoney DJ, Stojdl DF. Potentiating oncolytic viruses by targeted drug intervention. Curr Opin Mol Ther. 2010;12(4):394-402.

11. Rowan K. Oncolytic viruses move forward in clinical trials. $J$ Natl Cancer Inst. 2010;102(9):590-595.

12. Harrington KJ, Hingorani M, Tanay MA, et al. Phase I/II study of oncolytic HSV GM-CSF in combination with radiotherapy and cisplatin in untreated stage III/IV squamous cell cancer of the head and neck. Clin Cancer Res. 2010;16(15):4005-4015.

13. Kaufman HL, Bines SD. OPTIM trial: a phase III trial of an oncolytic herpes virus encoding GM-CSF for unresectable stage III or IV melanoma. Future Oncol. 2010;6(6):941-949.

14. Heo J, Breitbach CJ, Moon A, et al. Sequential therapy with JX-594, a targeted oncolytic poxvirus, followed by sorafenib in hepatocellular carcinoma: preclinical and clinical demonstration of combination efficacy. Mol Ther. 2011;19(6):1170-1179.

15. Breitbach CJ, Burke J, Jonker D, et al. Intravenous delivery of a multimechanistic cancer-targeted oncolytic poxvirus in humans. Nature. 2011;477(7362):99-102.

16. Yu W, Fang H. Clinical trials with oncolytic adenovirus in China. Curr Cancer Drug Targets. 2007;7(2):141-148.
17. Stanford MM, Breitbach CJ, Bell JC, McFadden G. Innate immunity, tumor microenvironment and oncolytic virus therapy: friends or foes? Curr Opin Mol Ther. 2008;10(1):32-37.

18. Wojton J, Kaur B. Impact of tumor microenvironment on oncolytic viral therapy. Cytokine Growth Factor Rev. 2010;21(2-3):127-134.

19. De Silva N, Atkins H, Kirn DH, Bell JC, Breitbach CJ. Double trouble for tumours: exploiting the tumour microenvironment to enhance anticancer effect of oncolytic viruses. Cytokine Growth Factor Rev. 2010;21(2-3):135-141.

20. Hay JG. The potential impact of hypoxia on the success of oncolytic virotherapy. Curr Opin Mol Ther. 2005;7(4):353-358.

21. Prestwich RJ, Errington F, Diaz RM, et al. The case of oncolytic viruses versus the immune system: waiting on the judgment of Solomon. Hum Gene Ther. 2009;20(10):1119-1132.

22. Boisgerault N, Tangy F, Gregoire M. New perspectives in cancer virotherapy: bringing the immune system into play. Immunotherapy. 2010;2(2):185-199.

23. Ottolino-Perry K, Diallo JS, Lichty BD, Bell JC, McCart JA. Intelligent design: combination therapy with oncolytic viruses. Mol Ther. 2010;18(2):251-263.

24. Roberts MS, Lorence RM, Groene WS, Bamat MK. Naturally oncolytic viruses. Curr Opin Mol Ther. 2006;8(4):314-321.

25. Obuchi M, Fernandez M, Barber GN. Development of recombinant vesicular stomatitis viruses that exploit defects in host defense to augment specific oncolytic activity. J Virol. 2003;77(16): $8843-8856$

26. Stojdl DF, Lichty BD, tenOever BR, et al. VSV strains with defects in their ability to shutdown innate immunity are potent systemic anticancer agents. Cancer Cell. 2003;4(4):263-275.

27. Wang F, Ma Y, Barrett JW, et al. Disruption of Erk-dependent type I interferon induction breaks the myxoma virus species barrier. Nat Immunol. 2004;5(12):1266-1274.

28. Mansour M, Palese P, Zamarin D. Oncolytic specificity of Newcastle disease virus is mediated by selectivity for apoptosis-resistant cells. J Virol. 2011;85(12):6015-6023.

29. Bauzon M, Hermiston TW. Oncolytic viruses: the power of directed evolution. Adv Virol. 2012; article no. 586389. doi:10.1155/2012/ 586389.

30. Kuhn I, Harden P, Bauzon M, et al. Directed evolution generates a novel oncolytic virus for the treatment of colon cancer. PLoS One. 2008;3(6):e2409.

31. Hanahan D, Weinberg RA. Hallmarks of cancer: the next generation. Cell. 2011;144(5):646-674.

32. Vaupel P, Kallinowski F, Okunieff P. Blood flow, oxygen and nutrient supply, and metabolic microenvironment of human tumors: a review. Cancer Res. 1989;49(23):6449-6465.

33. Whiteside TL. The tumor microenvironment and its role in promoting tumor growth. Oncogene. 2008;27(45):5904-5912.

34. Kurozumi K, Hardcastle J, Thakur R, et al. Effect of tumor microenvironment modulation on the efficacy of oncolytic virus therapy. $J$ Natl Cancer Inst. 2007;99(23):1768-1781.

35. Hockel M, Vaupel P. Tumor hypoxia: definitions and current clinical, biologic, and molecular aspects. J Natl Cancer Inst. 2001;93(4): 266-276.

36. Martinive P, Defresne F, Bouzin C, et al. Preconditioning of the tumor vasculature and tumor cells by intermittent hypoxia: implications for anticancer therapies. Cancer Res. 2006;66(24):11736-11744.

37. Matsumoto S, Yasui H, Mitchell JB, Krishna MC. Imaging cycling tumor hypoxia. Cancer Res. 2010;70(24):10019-10023.

38. Lin Q, Cong X, Yun Z. Differential hypoxic regulation of hypoxia-inducible factors 1alpha and 2alpha. Mol Cancer Res. 2011; 9(6):757-765.

39. Wenger RH. Mammalian oxygen sensing, signalling and gene regulation. J Exp Biol. 2000;203(Pt 8):1253-1263.

40. Pouyssegur J, Dayan F, Mazure NM. Hypoxia signalling in cancer and approaches to enforce tumour regression. Nature. 2006;441(7092): $437-443$. 
41. Wouters BG, Koritzinsky M. Hypoxia signalling through mTOR and the unfolded protein response in cancer. Nat Rev Cancer. 2008;8(11): 851-864.

42. Soeda A, Park M, Lee D, et al. Hypoxia promotes expansion of the CD133-positive glioma stem cells through activation of HIF-1alpha. Oncogene. 2009;28(45):3949-3959.

43. Hashimoto O, Shimizu K, Semba S, et al. Hypoxia induces tumor aggressiveness and the expansion of CD133-positive cells in a hypoxiainducible factor- $1 \alpha$-dependent manner in pancreatic cancer cells. Pathobiology. 2011;78(4):181-192.

44. Heddleston JM, Wu Q, Rivera M, et al. Hypoxia-induced mixed-lineage leukemia 1 regulates glioma stem cell tumorigenic potential. Cell Death Differ. August 12, 2011. [Epub ahead of print.] doi:10.1038/ cdd.2011.109.

45. Zhou Y, Zhou Y, Shingu T, et al. Metabolic alterations in highly tumorigenic glioblastoma cells: preference for hypoxia and high dependency on glycolysis. J Biol Chem. 2011;286(37):32843-32853.

46. Matsumoto K, Arao T, Tanaka K, et al. mTOR signal and hypoxiainducible factor-1 alpha regulate CD133 expression in cancer cells. Cancer Res. 2009;69(18):7160-7164.

47. Gardner LB, Li Q, Park MS, Flanagan WM, Semenza GL, Dang CV. Hypoxia inhibits $\mathrm{G} 1 / \mathrm{S}$ transition through regulation of $\mathrm{p} 27$ expression. J Biol Chem. 2001;276(11):7919-7926.

48. Box AH, Demetrick DJ. Cell cycle kinase inhibitor expression and hypoxia-induced cell cycle arrest in human cancer cell lines. Carcinogenesis. 2004;25(12):2325-2335.

49. Olcina M, Lecane PS, Hammond EM. Targeting hypoxic cells through the DNA damage response. Clin Cancer Res. 2010;16(23):5624-5629.

50. Young SD, Marshall RS, Hill RP. Hypoxia induces DNA overreplication and enhances metastatic potential of murine tumor cells. Proc Natl Acad Sci USA. 1988;85(24):9533-9537.

51. Rius J, Guma M, Schachtrup C, et al. NF-kappaB links innate immunity to the hypoxic response through transcriptional regulation of HIF-1alpha. Nature. 2008;453(7196):807-811.

52. Corzo CA, Condamine T, Lu L, et al. HIF-1alpha regulates function and differentiation of myeloid-derived suppressor cells in the tumor microenvironment. J Exp Med. 2010;207(11):2439-2453.

53. Bertout JA, Patel SA, Simon MC. The impact of $\mathrm{O} 2$ availability on human cancer. Nat Rev Cancer. 2008;8(12):967-975.

54. Imtiyaz HZ, Williams EP, Hickey MM, et al. Hypoxia-inducible factor 2alpha regulates macrophage function in mouse models of acute and tumor inflammation. J Clin Invest. 2010;120(8):2699-2714.

55. Sun X, Cheng G, Hao M, et al. CXCL12/CXCR4/CXCR7 chemokine axis and cancer progression. Cancer Metastasis Rev. 2010;29(4): 709-722.

56. Doedens AL, Stockmann C, Rubinstein MP, et al. Macrophage expression of hypoxia-inducible factor-1 alpha suppresses T-cell function and promotes tumor progression. Cancer Res. 2010;70(19) 7465-7475.

57. Lewis C, Murdoch C. Macrophage responses to hypoxia: implications for tumor progression and anti-cancer therapies. Am J Pathol. 2005;167(3):627-635.

58. Knowles HJ, Harris AL. Macrophages and the hypoxic tumour microenvironment. Front Biosci. 2007;12:4298-4314.

59. Mantovani A, Sica A. Macrophages, innate immunity and cancer: balance, tolerance, and diversity. Curr Opin Immunol. 2010;22(2):231-237.

60. Sitkovsky MV, Kjaergaard J, Lukashev D, Ohta A. Hypoxiaadenosinergic immunosuppression: tumor protection by $\mathrm{T}$ regulatory cells and cancerous tissue hypoxia. Clin Cancer Res. 2008;14(19): 5947-5952.

61. Noman MZ, Buart S, Van Pelt J, et al. The cooperative induction of hypoxia-inducible factor-1 alpha and STAT3 during hypoxia induced an impairment of tumor susceptibility to CTL-mediated cell lysis. J Immunol. 2009;182(6):3510-3521.

62. Hasmim M, Noman MZ, Lauriol J, et al. Hypoxia-dependent inhibition of tumor cell susceptibility to CTL-mediated lysis involves NANOG induction in target cells. J Immunol. 2011;187(8):4031-4039.
63. Facciabene A, Peng X, Hagemann IS, et al. Tumour hypoxia promotes tolerance and angiogenesis via CCL28 and T(reg) cells. Nature. 2011; 475(7355):226-230.

64. Wilson WR, Hay MP. Targeting hypoxia in cancer therapy. Nat Rev Cancer. 2011;11(6):393-410.

65. Dewhirst MW, Cao Y, Moeller B. Cycling hypoxia and free radicals regulate angiogenesis and radiotherapy response. Nat Rev Cancer. 2008; $8(6): 425-437$.

66. Rockwell S, Dobrucki IT, Kim EY, Marrison ST, Vu VT. Hypoxia and radiation therapy: past history, ongoing research, and future promise. Curr Mol Med. 2009;9(4):442-458.

67. Rohwer N, Cramer T. Hypoxia-mediated drug resistance: novel insights on the functional interaction of HIFs and cell death pathways. Drug Resist Updat. 2011;14(3):191-201.

68. Rohwer N, Dame C, Haugstetter A, et al. Hypoxia-inducible factor lalpha determines gastric cancer chemosensitivity via modulation of p53 and NF-kappaB. PLoS One. 2010;5(8):e12038.

69. Brown JM, Wilson WR. Exploiting tumour hypoxia in cancer treatment. Nat Rev Cancer. 2004;4(6):437-447.

70. Kottke T, Hall G, Pulido J, et al. Antiangiogenic cancer therapy combined with oncolytic virotherapy leads to regression of established tumors in mice. J Clin Invest. 2010;120(5):1551-1560.

71. Breitbach CJ, De Silva NS, Falls TJ, et al. Targeting tumor vasculature with an oncolytic virus. Mol Ther. 2011;19(5):886-894.

72. Tseng JC, Granot T, DiGiacomo V, Levin B, Meruelo D. Enhanced specific delivery and targeting of oncolytic Sindbis viral vectors by modulating vascular leakiness in tumor. Cancer Gene Ther. 2010; 17(4):244-255.

73. Roulston A, Marcellus RC, Branton PE. Viruses and apoptosis. Annu Rev Microbiol. 1999;53:577-628.

74. Breitbach CJ, Paterson JM, Lemay CG, et al. Targeted inflammation during oncolytic virus therapy severely compromises tumor blood flow. Mol Ther. 2007;15(9):1686-1693.

75. Kirn DH, Wang Y, Le Boeuf F, Bell J, Thorne SH. Targeting of interferon-beta to produce a specific, multi-mechanistic oncolytic vaccinia virus. PLoS Med. 2007;4(12):e353.

76. Hernandez-Alcoceba R, Pihalja M, Qian D, Clarke MF. New oncolytic adenoviruses with hypoxia- and estrogen receptor-regulated replication. Hum Gene Ther. 2002;13(14):1737-1750.

77. Post DE, Van Meir EG. A novel hypoxia-inducible factor (HIF) activated oncolytic adenovirus for cancer therapy. Oncogene. 2003; 22(14):2065-2072.

78. Cuevas Y, Hernandez-Alcoceba R, Aragones J, et al. Specific oncolytic effect of a new hypoxia-inducible factor-dependent replicative adenovirus on von Hippel-Lindau-defective renal cell carcinomas. Cancer Res. 2003;63(20):6877-6884.

79. Cho WK, Seong YR, Lee YH, et al. Oncolytic effects of adenovirus mutant capable of replicating in hypoxic and normoxic regions of solid tumor. Mol Ther. 2004;10(5):938-949.

80. Post DE, Devi NS, Li Z, et al. Cancer therapy with a replicating oncolytic adenovirus targeting the hypoxic microenvironment of tumors. Clin Cancer Res. 2004;10(24):8603-8612.

81. Shen BH, Hermiston TW. Effect of hypoxia on Ad5 infection, transgene expression and replication. Gene Ther. 2005;12(11):902-910.

82. Pipiya T, Sauthoff H, Huang YQ, et al. Hypoxia reduces adenoviral replication in cancer cells by downregulation of viral protein expression. Gene Ther. 2005;12(11):911-917.

83. Shen BH, Bauzon M, Hermiston TW. The effect of hypoxia on the uptake, replication and lytic potential of group B adenovirus type 3 (Ad3) and type 11p (Ad11p). Gene Ther. 2006;13(12):986-990.

84. Cherry T, Longo SL, Tovar-Spinoza Z, Post DE. Second-generation HIF-activated oncolytic adenoviruses with improved replication, oncolytic, and antitumor efficacy. Gene Ther. 2010;17(12):1430-1441.

85. Zhang Q, Chen G, Peng L, et al. Increased safety with preserved antitumoral efficacy on hepatocellular carcinoma with dualregulated oncolytic adenovirus. Clin Cancer Res. 2006;12(21): 6523-6531. 
86. Wang X, Su C, Cao H, et al. A novel triple-regulated oncolytic adenovirus carrying p53 gene exerts potent antitumor efficacy on common human solid cancers. Mol Cancer Ther. 2008;7(6):1598-1603.

87. Harvey TJ, Hennig IM, Shnyder SD, et al. Adenovirus-mediated hypoxia-targeted gene therapy using HSV thymidine kinase and bacterial nitroreductase prodrug-activating genes in vitro and in vivo. Cancer Gene Ther. 2011;18(11):773-784.

88. Guse K, Diaconu I, Rajecki M, et al. Ad5/3-9HIF-Delta24-VEGFR1 -Ig, an infectivity enhanced, dual-targeted and antiangiogenic oncolytic adenovirus for kidney cancer treatment. Gene Ther. 2009;16(8): 1009-1020.

89. Gomes EM, Rodrigues MS, Phadke AP, et al. Antitumor activity of an oncolytic adenoviral-CD40 ligand (CD154) transgene construct in human breast cancer cells. Clin Cancer Res. 2009;15(4):1317-1325.

90. Post DE, Sandberg EM, Kyle MM, et al. Targeted cancer gene therapy using a hypoxia inducible factor dependent oncolytic adenovirus armed with interleukin-4. Cancer Res. 2007;67(14):6872-6881.

91. Kwon OJ, Kim PH, Huyn S, et al. A hypoxia- and \{alpha\}-fetoproteindependent oncolytic adenovirus exhibits specific killing of hepatocellular carcinomas. Clin Cancer Res. 2010;16(24):6071-6082.

92. Aghi MK, Liu TC, Rabkin S, Martuza RL. Hypoxia enhances the replication of oncolytic herpes simplex virus. Mol Ther. 2009;17(1): 51-56.

93. Fasullo M, Burch AD, Britton A. Hypoxia enhances the replication of oncolytic herpes simplex virus in p53- breast cancer cells. Cell Cycle. 2009;8(14):2194-2197.

94. Longo SL, Griffith C, Glass A, Shillitoe EJ, Post DE. Development of an oncolytic herpes simplex virus using a tumor-specific HIF-responsive promoter. Cancer Gene Ther. 2011;18(2):123-134.

95. Hiley CT, Yuan M, Lemoine NR, Wang Y. Lister strain vaccinia virus, a potential therapeutic vector targeting hypoxic tumours. Gene Ther. 2010;17(2):281-287.

96. Connor JH, Naczki C, Koumenis C, Lyles DS. Replication and cytopathic effect of oncolytic vesicular stomatitis virus in hypoxic tumor cells in vitro and in vivo. J Virol. 2004;78(17):8960-8970.

97. Hwang II, Watson IR, Der SD, Ohh M. Loss of VHL confers hypoxiainducible factor (HIF)-dependent resistance to vesicular stomatitis virus: role of HIF in antiviral response. J Virol. 2006;80(21):10712-10723.

98. Maxwell PH, Wiesener MS, Chang GW, et al. The tumour suppressor protein VHL targets hypoxia-inducible factors for oxygen-dependent proteolysis. Nature. 1999;399(6733):271-275.

99. Willmon C, Diaz RM, Wongthida P, et al. Vesicular stomatitis virus-induced immune suppressor cells generate antagonism between intratumoral oncolytic virus and cyclophosphamide. Mol Ther. 2011;19(1):140-149.
100. Leveille S, Goulet ML, Lichty BD, Hiscott J. VSV oncolytic treatment interferes with tumor associated dendritic cell function and abrogates tumor antigen presentation. J Virol. 2011;85(23): 12160-12169.

101. Cho IR, Koh SS, Min HJ, et al. Down-regulation of HIF-1alpha by oncolytic reovirus infection independently of VHL and p53. Cancer Gene Ther. 2010;17(5):365-372.

102. Rudin CM, Poirier JT, Senzer NN, et al. Phase I clinical study of Seneca Valley Virus (SVV-001), a replication-competent picornavirus, in advanced solid tumors with neuroendocrine features. Clin Cancer Res. 2011;17(4):888-895.

103. Roos FC, Roberts AM, Hwang II, et al. Oncolytic targeting of renal cell carcinoma via encephalomyocarditis virus. EMBO Mol Med. 2010;2(7):275-288.

104. Murdoch C, Giannoudis A, Lewis CE. Mechanisms regulating the recruitment of macrophages into hypoxic areas of tumors and other ischemic tissues. Blood. 2004;104(8):2224-2234.

105. Qian BZ, Pollard JW. Macrophage diversity enhances tumor progression and metastasis. Cell. 2010;141(1):39-51.

106. Muthana M, Giannoudis A, Scott SD, et al. Use of macrophages to target therapeutic adenovirus to human prostate tumors. Cancer Res. 2011;71(5):1805-1815.

107. Baas T. Hypoxic homing in prostate cancer. SciBX. 2011; March 24. doi:10.1038/scibx.2011.1330.

108. Kumar S, Gao L, Yeagy B, Reid T. Virus combinations and chemotherapy for the treatment of human cancers. Curr Opin Mol Ther. 2008;10(4):371-379.

109. Owen MR, Stamper IJ, Muthana M, et al. Mathematical modeling predicts synergistic antitumor effects of combining a macrophagebased, hypoxia-targeted gene therapy with chemotherapy. Cancer Res. 2011;71(8):2826-2837.

110. Prestwich RJ, Harrington KJ, Pandha HS, et al. Oncolytic viruses: a novel form of immunotherapy. Expert Rev Anticancer Ther. 2008; 8(10):1581-1588.

111. Melcher A, Parato K, Rooney CM, Bell JC. Thunder and lightning: immunotherapy and oncolytic viruses collide. Mol Ther. 2011; 19(6):1008-1016.

112. Pol JG, Resseguier J, Lighty BD. Oncolytic viruses, a step into cancer immunotherapy. Virus Adapt Treat. 2011;3. In press.

113. Carmeliet P, Jain RK. Principles and mechanisms of vessel normalization for cancer and other angiogenic diseases. Nat Rev Drug Discov. 2011;10(6):417-427.

114. Qayum N, Muschel RJ, Im JH, et al. Tumor vascular changes mediated by inhibition of oncogenic signaling. Cancer Res. 2009;69(15): 6347-6354.
Virus Adaptation and Treatment

\section{Publish your work in this journal}

Virus Adaptation and Treatment is an international, peer-reviewed open access journal focusing on the study of virology, viral adaptation and the development and use of antiviral drugs and vaccines to achieve improved outcomes in infection control and treatment. The journal welcomes original research, basic science, clinical \& epidemiological

\section{Dovepress}

studies, reviews \& evaluations, expert opinion and commentary, case reports and extended reports. The manuscript management system is completely online and includes a very quick and fair peer-review system, which is all easy to use. Visit http://www.dovepress.com/ testimonials.php to read real quotes from published authors. 